\title{
Low dose metronomic oral cyclophosphamide for hormone resistant prostate cancer: a phase II study
}

R Lord, S Nair*, A Schache **, J Spicer, Navita Somaihah, V Khoo *** and H Pandha ${ }^{\dagger}$.

Department of Oncology and Urology* St George's, University of London, Cranmer Terrace, London SW17 ORE, United Kingdom

**Department of Urology, Frimley Park Hospital,

***Academic Department of Urology, Royal Marsden Hospital, Sutton, Surrey, UK SM2 5PT

$\dagger$ Department of Oncology, Postgraduate Medical School, University of Surrey, Daphne Jackson Road, Guildford, GU2 7WG

Running title: Low dose metronomic oral cyclophosphamide.

Correspondence to Prof Hardev Pandha E mail h.pandha@surrey.ac.uk Tel 01483688550

Fax 01483688558

Keywords: cyclophosphamide, prostate, cancer, hormone refractory, metronomic. 


\section{$\underline{\text { Abstract }}$}

Purpose: Cyclophosphamide (CY) is a bifunctional alkylating agent long associated with immune activation. Continuous uninterrupted low (so-called metronomic) doses of CY can lead to enhanced immunity against a variety of antigens possibly by targeting regulatory $\mathrm{T}$ cells ( $\mathrm{T}$ reg) and/or tumour angiogenesis. In this study we tested the observations from animal models and evaluated the safety and efficacy of continuous low dose oral CY in patients with HRPC.

Materials and Methods: Eighty patients were recruited over a 2 year period and fifty eight received at least 2 cycles ( 8 weeks) of $50 \mathrm{mg} / \mathrm{m}^{2}$ oral $\mathrm{CY}$ to be included in the safety and intention-to-treat analysis.

Results: Metronomic CY was safe and well tolerated, and although lymphopaenia (up to grade 3) was observed in a third of all patients, there were no clinical complications. The response rate was $34.5 \%$ inclusive of objective and PSA (absolute reduction and reduction in PSA velocity). The median duration of response was 7.5 months (range 318 months).

Conclusions: Oral CY can be used on a metronomic basis safely in men with HRPC. The efficacy, low toxicity, low cost and ease of administration of CY justifies further studies in prostate cancer in combination with other agents. 


\section{Introduction}

Despite recent progress in the management of early and localised prostate cancer (PC), a substantial number of patients still develop hormone resistant (HRPC) and metastatic disease that can significantly impact on symptoms, quality of life and longevity ${ }^{1}$. There has been considerable interest in exploiting the immunostimulatory and antiangiogenic potential of drugs previously thought to be beneficial solely in terms of their cytotoxicity $^{2}$. Cyclophosphamide (CY) is a bifunctional alkylating agent which has been used to treat a very large range of malignancies and has also been long associated with immune activation ${ }^{3}$, and more recently with anti-angiogenesis. Low doses of CY can lead to enhanced immune responses against a variety of antigens. In murine models, low dose CY decreases numbers of suppressor or regulatory $\mathrm{T}$ cells (T reg) and directly inhibits the suppressive capability of CD4 $+25+\mathrm{T}$ reg cells by enhancing apoptosis and decreasing homeostatic proliferation. Higher doses of CY are associated with cytotoxicity and immunosuppression, whereas continuous low dose scheduling may be immunostimulatory and/or antiangiogenic ${ }^{4}$. Metronomic dosing involves the chronic administration of comparatively low doses of $\mathrm{CY}$ at close, regular intervals, with no prolonged drug-free interruptions ${ }^{5}$. This approach is not necessarily designed with the intention of substantially increasing cumulative drug-dose over time; as such it minimizes host toxic side effects and the need for supportive care treatment. Metronomic dosing of agents such as CY or trofosfamide has shown surprisingly potent and durable antitumour effects in a number of models, including transplantable human lymphoma $^{6}$ and human prostate carcinoma ${ }^{7}$. 
In this study we have evaluated the safety and efficacy of continuous low dose oral cyclophophamide in patients with HRPC, both in view of its potential as a cost effective single agent to palliate HRPC but also possibly as a synergistic agent with cancer vaccines or other antiangiogenic agents. 


\section{Materials and methods}

\section{Eligibility Criteria}

Men with histologically confirmed prostate cancer, who failed optimal first line androgen deprivation therapy with leutenizing hormone releasing hormone (LHRH) agonist drugs, total androgen blockade therapy or orchidectomy were eligible for the trial. Patients were required to have progressive disease as indicated by 2 consecutive rises in Prostate Specific Antigen (PSA), separated by at least four weeks, from a minimum level of $2 \mathrm{ng} / \mathrm{ml}$; WHO performance status of $0-2$ and a life expectancy of at least six months. Biochemical trial entry parameters included castrate levels of serum testosterone $(<50 \mathrm{ng} / \mathrm{ml})$, adequate bone marrow function, as defined by white blood count of more than $3500 / \mathrm{mm}^{3}$, haemoglobin of more than $10 \mathrm{~g} / \mathrm{dl}$, platelet count of at least $100000 / \mathrm{mm}^{3}$; adequate renal function with serum creatinine of less than $2.0 \mathrm{mg} / \mathrm{dl}$ and adequate hepatic function defined as less than 2 times upper limit of normal value for aspartate transaminase and alanine transaminase. Patients continued their LHRH agonist and/or antiandrogen therapy to avoid altered PSA kinetics associated with androgen withdrawal. All other treatments were discontinued. Patients with visceral and brain metastases were excluded. All patients provided written informed consent. The study was approved by St Georges Hospital Local Research Ethics Committee.

\section{Trial design}

This was a single centre open label phase II study for patients with HRPC. Patients with hormone resistant rather than hormone refractory disease were selected on the basis that the addition of casodex withdrawal as a treatment does not alter the natural history of 
the disease overall. Patients received oral cyclophosphamide $50 \mathrm{mg} / \mathrm{m}^{2}$ continuously in 4 weekly cycles without breaks, for a total of 12 cycles. Further treatment was allowed if only grade 1 or 2 toxicity had occurred. Adverse events were graded according to NCI Common Toxicity Criteria (CTC) version II. All patients underwent assessment of tumour status at baseline, six months and one year or at the time of withdrawal, using computed tomography (CT) and technicium-99 bone scan. Clinical examination, PSA monitoring, biochemical and haematological profiles were performed in all patients at the beginning of the study and at each 4 weekly visit. Patients were withdrawn from the study whenever any of the following occurred: PSA increase of $25 \%$ over baseline (or nadir if responding initially) after a minimum of 3 cycles and corroborated by a repeat PSA test within 2 weeks, disease progression (defined by Response Evaluation Criteria In Solid Tumours (RECIST) criteria), worsening of PC-related symptoms; patient initiated withdrawal for any reason; development of intercurrent illness requiring treatment with corticosteroids; development of any severe (> grade 3) toxicity or lack of compliance with the protocol. Objective responses were defined according to RECIST criteria.

\section{PSA Velocity}

PSAV was calculated for each patient pre-treatment and post-treatment, by fitting a regression model to the $\log$ of the PSA values. The model used was $\ln (\mathrm{PSA}) / \mathrm{t}$, where $t$ is the time between enrolment on the trial and the date of the PSA sample. PSA doubling time was calculated for each patient as $\ln (2) /$ PSA Velocity. 


\section{Study objectives and assessment of response.}

The primary endpoint of this study was PSA response (50\% fall in PSA levels sustained for at least 4 weeks) as defined by PSA working group criteria. Secondary endpoints included proportion of evaluable patients who showed complete or partial objective radiological response to treatment, a fall in absolute PSA or a fall in PSA velocity, duration of any responses and the safety and tolerability of the continuous dosing. Measurement of serum markers of angiogenesis and enumeration and functional analysis of peripheral blood Treg was completed in a subset of patients. The study was a two stage design using the Gehan principle ${ }^{8}$. Initially 15 patients were recruited, then extended to 50 patients as responses were observed in at least 2 individuals. The study was further extended to 80 patients after discussion with the LREC. 


\section{$\underline{\text { Results }}$}

\section{Patient Characteristics}

A total of 80 patients were recruited over a 2 year period (from February 2002 to February 2004). Fifty eight received at least 2 cycles (8 weeks) of study medication and were included in the safety and intention-to-treat analysis (ITT). The median duration of treatment for these patients was 8 cycles. Eighteen patients received less than 8 weeks of therapy and therefore were non-evaluable. This was due to patient choice (2/18 cases), rapidly progressive symptoms (7/18), rapid PSA rise (8/18) and rash (1/18). The remaining four patients were ineligible as they commenced other medication which may have affected the PSA value. Mean age of the patients was 70 years (range 51-86); $78 \%$ of patients were Caucasian and $22 \%$ Afro-Caribbean. The WHO performance status was $0,1,2$ in $16(20 \%), 28(35 \%)$ and $36(45 \%)$ of the patients respectively, table 1 . The median PSA was 43.9 and 168 for the evaluable and non-evaluable cohorts respectively. The mean combined Gleason grade was 7 (range 69) in evaluable and 8 in non-evaluable (range 6-10) patients. Over half of the patients had localised recurrent disease characterised by a normal bone scan, absence of nodal or visceral metastases but with a rising PSA despite LHRH-containing hormonal therapy.

\section{Toxicity}

Toxicity information was collected for all patients. For each patient, the worst grades seen of the most common haematologic and non-haematologic toxicities are summarized in table 2. The treatment was generally well tolerated. Grade 2 fatigue symptoms were reported in five patients after 6 months, although all patients chose to 
continue with the treatment. The study agent did not exacerbate urinary symptoms in any patient, nor was there evidence of haematuria on urine testing, even after prolonged administration. The most significant finding was grade 3 lymphopaenia, observed in 19 $(32.8 \%)$ patients. Lymphopaenia is not normally a limiting toxicity in studies involving chemotherapeutic agents. This persisted despite dose reducing or stopping the drug in 3 patients, and necessitated prophylactic cover with oral co-trimoxazole for opportunistic infection, and conversion to a lower dose or alternative therapy. In total five patients had dose reductions to their therapy. No clinical or radiological indications of an opportunistic infection as a result of lymphopaenia were found at any time. Three patients required treatment breaks of over 2 weeks before re-commencing at $25 \%$ dose reduction due to neutropaenia, anaemia and diarrhoea. One patient developed a sputum culture-positive bacterial respiratory infection which responded promptly to amoxycillin. A further patient developed a typical allergic drug rash after the second day of treatment. Two patients developed non-painful cutaneous herpes zoster after 4 and 6 months, which responded to zovirax and had no subsequent sequelae. No second malignancy has been reported in any study patient to date.

\section{Efficacy}

On an intention to treat basis, the response rate was $34.5 \%$ inclusive of objective and PSA (absolute reduction and reduction in PSAV) parameters, table 3. One patient had a partial response by RECIST, and two patients a PSA fall of $>50 \%$. The majority of the responders had localised disease, with no clear difference in patients with bone and/or nodal disease. There was no correlation between response and Gleason grading or ethnicity. The majority of the non responders had either locally advanced or bone 
metastatic disease. The median duration of response was 7.5 months (confidence intervals 3-18 months), the median survival has not yet been reached. The overall survival is shown in figure 1 . The proportion of patients still alive reflects the high proportion with localised disease at recruitment. There was no correlation between survival and Gleason grading or site of metastases. The majority of patients stopped treatment due to rapidly rising PSA, although a significant proportion included grade 3 haematological toxicity (lymphopaenia mainly) or objective progression by radiology, table 4. Four patients continued with therapy beyond the first 12 cycles. 


\section{$\underline{\text { Discussion }}$}

Despite the reported survival benefit with taxane-based chemotherapy, the prognosis for HRPC patients is still guarded. This study has shown that CY may be administered in a metronomic schedule and is capable of positively altering PSA kinetics without significant toxicity. The median duration of response to metronomic CY was at least that expected with second line hormonal agents but without the toxicity. The advantages of low dose CY were its convenient oral administration, low cost, dosing schedule and lack of toxicity.

The patients enrolled to the study represented a typical cross section of this disease in the clinic- including patients with purely biochemical PSA failure up to those with more advanced and evaluable disease. The majority of patients had localised disease and rising PSA although significant numbers of patients with bone metastases were included. Our experience with immunological status of patients with nodal (or no) metastases versus bone metastases indicates the presence of bone disease is associated with profound immunosuppression (manuscript submitted). Patients who were unable to continue with the study to the 3 month time point so as to be non-evaluable were characterised by a higher mean PSA, more bone or bone/nodal disease and a slightly higher Gleason grade of 8 .

Treatment related toxicity was mild, with no CY-related serious adverse events and no symptoms to suggest haemorrhagic cystitis or second malignancies. Although lymphopaenia was observed in 19 patients, there were no clinical complications. Myelotoxicity has been reported with this dose of CY previously, and it is also possible that a significant proportion of men had disease metastatic to bone marrow and/or anaemia of chronic disease. 
Although the number of responders was modest on conventional criteria, there have been a number of studies indicating the utility of PSA kinetics as response criteria. This has included reduction in PSA of less than 50\% and reduction in PSA velocity (increased PSA doubling time) ${ }^{9}$. On the other hand, vaccine therapy for prostate cancer has been associated with increased survival without significant alterations in serum $\mathrm{PSA}^{10}$. In our recent evaluation of an allogeneic whole cell vaccine for HRPC patients, none of the 28 patients enrolled had objective or conventional PSA responses but a significant proportion had a fall in PSA velocity and an increased time to disease progression compared to other agents used for the same patient group ${ }^{11}$. In terms of the 32 non-responders in this study, there were equal number of patients with bone involved and locally advanced tumours. There was no clear correlation with their histology, ethnicity or previous therapy. It is noteworthy that the main reason to stop treatment in these patients was related to disease progression as noted by an increase in PSA levels, worsening radiological or clinical symptoms and not due to toxicity of the agent.

It is clear from murine and human studies that the biological effect of CY may depend on dose and delivery schedule. Higher doses are associated with cytotoxicity and immunosuppression, whereas continuous low dose scheduling may be immunostimulatory and/or antiangiogenic. Previous studies have reported significant stabilising effects of CY but modest if any response to moderate to high doses of bolus CY, although these studies are from the pre-PSA and pre-CT scan era ${ }^{12}$. Metronomic type dosing of $\mathrm{CY}$ has been previously reported as a single agent in one 5 patient study ${ }^{13}$ but otherwise has been used in combination with potentially immunosuppressive agents such as steroids and conventional doses of cytotoxic agents in prostate and other cancers. We believe that the observed effects of CY were unlikely to be explained 
purely by the cytotoxic actions of $\mathrm{CY}$ at this dose. Instead, the immunoregulatory and anti-angiogenic effects were probably more likely to explain the data. We assessed the effect of continuous $\mathrm{CY}$ on circulating $\mathrm{T}$ regs in 20 patients after 3 months following numerous reports of effects in murine models and found no clear correlation with any clinical, pathological or biochemical factors in the study (data not shown). A comparison after 3 months may have been insufficient time, and changes in the intratumoural rather than the circulating $\mathrm{T}$ regs may be more important to ascertain. Although the number of samples available for analysis was ultimately small, we did observe a reduction in expression of TGF $\beta$ by the T regs post- versus pre-treatment. The antiangiogenic effects of CY are well documented, with direct evidence of effect on microvessel sprouting ${ }^{14}$. PC is associated with a large number of angiogenic factors, and VEGF expression is increased in androgen-independent tumours ${ }^{15}$. Hamano et al. (2004) demonstrated that low-dose cyclophosphamide inhibits tumour growth by upregulating the endogenous angiogenesis inhibitor thrombospondin-1 in tumour and perivascular cells. Thrombospondin-1, in turn, promotes endothelial cell apoptosis ${ }^{16}$. CY may be acting as an antiangiogenic agent in this study by induction of potent antiangiogenic factors such as thromobospondin in tumour cells ${ }^{10}$. Although our assessment of VEGF expression was inconclusive, there are a number of newer assays which may be utilised in future studies.

\section{Conclusions}

In summary, we have shown that oral CY can be used on a continual basis safely in men with HRPC. Antitumour and marker responses were observed in patients with nodal and bone metastatic disease. The low toxicity, low cost, ease of administration, immunoregulatory and antiangiogenic potential of CY makes it a prime candidate for 
combination with vaccines and other antiangiogenic agents. For the palliative effects shown in this study we intend to pursue a further study randomising metronomic CY against dethyl stilboestrol to include quality of life assessment. 


\section{Acknowledgements}

We thank our oncology and urology colleagues for referring patients for this study, and Prof Bernard Bonnotte for critically reading the manuscript. HP was Hon. Simon Weinstock Senior Lecturer in Tumour Immunology funded by the Sobel Trust.

\section{References}

1) Zhang Y, Glass A, Bennett N, Oyama KA, Gehan E, Gelmann EP. Long-term outcomes after radical prostatectomy performed in a community-based health maintenance organization. Cancer 2004; 100(2):300-7.

2) Albini A, Tosetti F, Benelli R, Noonan DM. Tumour inflammatory angiogenesis and its chemoprevention. Cancer Res 2005; 65(23):10637-41.

3) Lutsiak ME, Semnani RT, De Pascalis R, Kashmiri SV, Schlom J, Sabzevari H. Inhibition of $\mathrm{CD} 4(+) 25+\mathrm{T}$ regulatory cell function implicated in enhanced immune response by low-dose cyclophosphamide. Blood 2005; 105(7):2862-8.

4) Damber JE, Vallbo C, Albertsson P, Lennernas B, Norrby K. The anti-tumour effect of low-dose continuous chemotherapy may partly be mediated by thrombospondin. Cancer Chemother Pharmacol: 2005 1-7.

5) Hanahan D, Bergers G, Bergsland E. Less is more, regularly: metronomic dosing of cytotoxic drugs can target tumor angiogenesis in mice. J Clin Invest 2000; 105(8):10456) Browder T, Butterfield CE, Kraling BM, Shi B, Marshall B, O'Reilly MS, Folkman J. Antiangiogenic scheduling of chemotherapy improves efficacy against experimental drug-resistant cancer. Cancer Res 2000; 60(7):1878-86.

7) Man S, Bocci G, Francia G, Green SK, Jothy S, Hanahan D, Bohlen P, Hicklen DJ, Bergers G, Kerbel RS Antitumor effects in mice of low-dose (metronomic) 
cyclophosphamide administered continuously through the drinking water. Cancer Res 2002; 62(10):2731-5.

8) Gehan EA, (1979) Clinical Trials in Cancer Research. Environ Health Perspect. 32: $31-48$

9) D'Amico AV, Moul J, Carroll PR, Sun L, Lubeck D, Chen MH. Prostate specific antigen doubling time as a surrogate end point for prostate cancer specific mortality following radical prostatectomy or radiation therapy. J Urol 2004 172(5 Pt 2):S42-6; discussion S46-7.

10) Schellhammer PF, Hershberg RM. Immunotherapy with autologous antigen presenting cells for the treatment of androgen independent prostate cancer. World J Urol $200523(1): 47-9$.

11) Michael A, Ball G, Quatan N, Wushishi F, Russell N, Whelan J, Chakraborty P, Leader D, Whelan M, Pandha H Delayed disease progression after allogeneic cell vaccination in hormone-resistant prostate cancer and correlation with immunologic variables. Clin Cancer Res . 2005 11(12):4469-78.

12) Chlebowski RT, Hestorff R, Sardoff L, Weiner J, Bateman JR. Cyclophosphamide (NSC 26271) versus the combination of adriamycin (NSC 123127), 5-fluorouracil (NSC 19893), and cyclophosphamide in the treatment of metastatic prostatic cancer: a randomized trial. Cancer 1978 42(6):2546-52.

13) Nicolini A, Mancini P, Ferrari P, Anselmi L, Tartarelli G, Bonazzi V, Carpi A, Giardino R. Oral low-dose cyclophosphamide in metastatic hormone refractory prostate cancer (MHRPC). Biomed Pharmacother 2004 58(8):447-50.

14) Albertsson P, Lennernas B, Norrby K. Chemotherapy and antiangiogenesis: drugspecific effects on microvessel sprouting. Apmis 2003 111(11):995-1003 
15) Nicholson B, Theodorescu D. Angiogenesis and prostate cancer tumor growth. J Cell Biochem 2004 91(1):125-50.

16) Hamano Y, Sugimoto H, Soubasakos MA. et al. Thrombospondin-1 associated with tumor microenvironment contributes to low-dose cyclophosphamide-mediated endothelial cell apoptosis and tumor growth suppression. Cancer Res 2004 64(5):1570-4.

\section{Figure Legends}

Figure 1 - Overall survival of hormone resistant prostate cancer patients treated with low dose metronomic oral cyclophosphamide. 
Evaluable

$(\mathrm{n}=58)$
Non-Evaluable

$(\mathrm{n}=18)$

\begin{tabular}{|c|c|c|c|c|}
\hline \multicolumn{5}{|l|}{ Age, years } \\
\hline Range & $51-86$ & & $60-84$ & \\
\hline Median & 69 & & 71 & \\
\hline \multicolumn{5}{|l|}{ Baseline PSA } \\
\hline Range & $2.4-789$ & & $19-1973$ & \\
\hline Median & 43.9 & & 168 & \\
\hline Sites of Disease & number & $\%$ & number & $\%$ \\
\hline Localised & 30 & 51.7 & 2 & 11.1 \\
\hline Bone & 20 & 34.5 & 11 & 61.1 \\
\hline Nodal & 4 & 6.9 & 2 & 11.1 \\
\hline Bone \& Nodal & 4 & 6.9 & 3 & 16.6 \\
\hline & $1-24$ & & $1-2$ & \\
\hline *Number of & $2-21$ & & $2-6$ & \\
\hline previous & $3-9$ & & $3-8$ & \\
\hline treatments & $>3-1$ & & $>3-2$ & \\
\hline
\end{tabular}

*where previous therapy not confirmed, omitted

Table 1 Patient demographics. 


\begin{tabular}{|c|c|c|c|}
\hline \multicolumn{4}{|c|}{ СTC } \\
\hline Toxicity & Grade & $\mathbf{n}$ & $\%$ \\
\hline \multirow[t]{3}{*}{ Lymphopenia } & 1 & 3 & 5.2 \\
\hline & 2 & 36 & 62 \\
\hline & 3 & 19 & 32.8 \\
\hline \multirow[t]{3}{*}{ Neutropenia } & 1 & 4 & 6.9 \\
\hline & 2 & 1 & 1.7 \\
\hline & 3 & 1 & 1.7 \\
\hline \multirow[t]{3}{*}{ Thrombocytopenia } & 1 & 5 & 8.6 \\
\hline & 2 & 3 & 5.2 \\
\hline & 3 & 0 & 0 \\
\hline \multirow[t]{3}{*}{ Anaemia } & 1 & 3 & 5.2 \\
\hline & 2 & 12 & 20.7 \\
\hline & 3 & 1 & 1.7 \\
\hline \multirow[t]{2}{*}{ Fatigue } & 1 & 5 & 8.6 \\
\hline & 2 & 5 & 8.6 \\
\hline Nausea & 1 & 1 & 1.7 \\
\hline Renal impairment & 1 & 1 & 1.7 \\
\hline Diarrhoea & 1 & 1 & 1.7 \\
\hline Rash & 1 & 1 & 1.7 \\
\hline
\end{tabular}

Table 2 Toxicity observed in all evaluable patients using NCI Common Toxicity Criteria. 


\section{Sites of Disease (\%)}

Bone \&

\begin{tabular}{|c|c|c|c|c|c|c|}
\hline & $n=58$ & $\%$ & Local & Bone & Nodal & Nodal \\
\hline Responders & 26 & 44.8 & $14(54)$ & $5(19.2)$ & $4(15.4)$ & $3(11.5)$ \\
\hline \multicolumn{7}{|l|}{ Objective \& PSA } \\
\hline Responders & 3 & 5.2 & $1(33.3)$ & $1(33.3)$ & $0(0)$ & $1(33.3)$ \\
\hline \multicolumn{7}{|l|}{ PSA Responders } \\
\hline$(<50 \%)$ & 10 & 17.2 & $6(60)$ & $2(20)$ & $1(10)$ & $1(10)$ \\
\hline \multicolumn{7}{|l|}{ PSA Velocity } \\
\hline Responders & 13 & 22.4 & $7(54)$ & $2(15.4)$ & $3(23)$ & $1(7.7)$ \\
\hline Non-Responders & 32 & 55.2 & $16(50)$ & $15(46.9)$ & $0(0)$ & $1(3.1)$ \\
\hline Total & 58 & 100 & 30 & 20 & 4 & 4 \\
\hline
\end{tabular}

Table 3 Response to CY therapy and correlation to site of disease 


\begin{tabular}{|lcc|}
\multicolumn{1}{|l|}{\begin{tabular}{c} 
Reason for Stopping \\
\multicolumn{1}{|c|}{ Treatment }
\end{tabular}} & $\mathbf{n}=\mathbf{5 8}$ & $\mathbf{\%}$ \\
\hline Rising PSA & 29 & 50 \\
\hline Objective PD & 9 & 15.5 \\
\hline Progressive Symptoms & 2 & 3.5 \\
\hline Haematological Toxicity & 13 & 22.4 \\
\hline Duration of Therapy $>12$ & & \\
months & 4 & 6.9 \\
\hline Treatment Ongoing & & \\
\hline
\end{tabular}

Table 4 Treatment status of patients and reasons for stopping metronomic CY therapy 


\section{Survival Function}

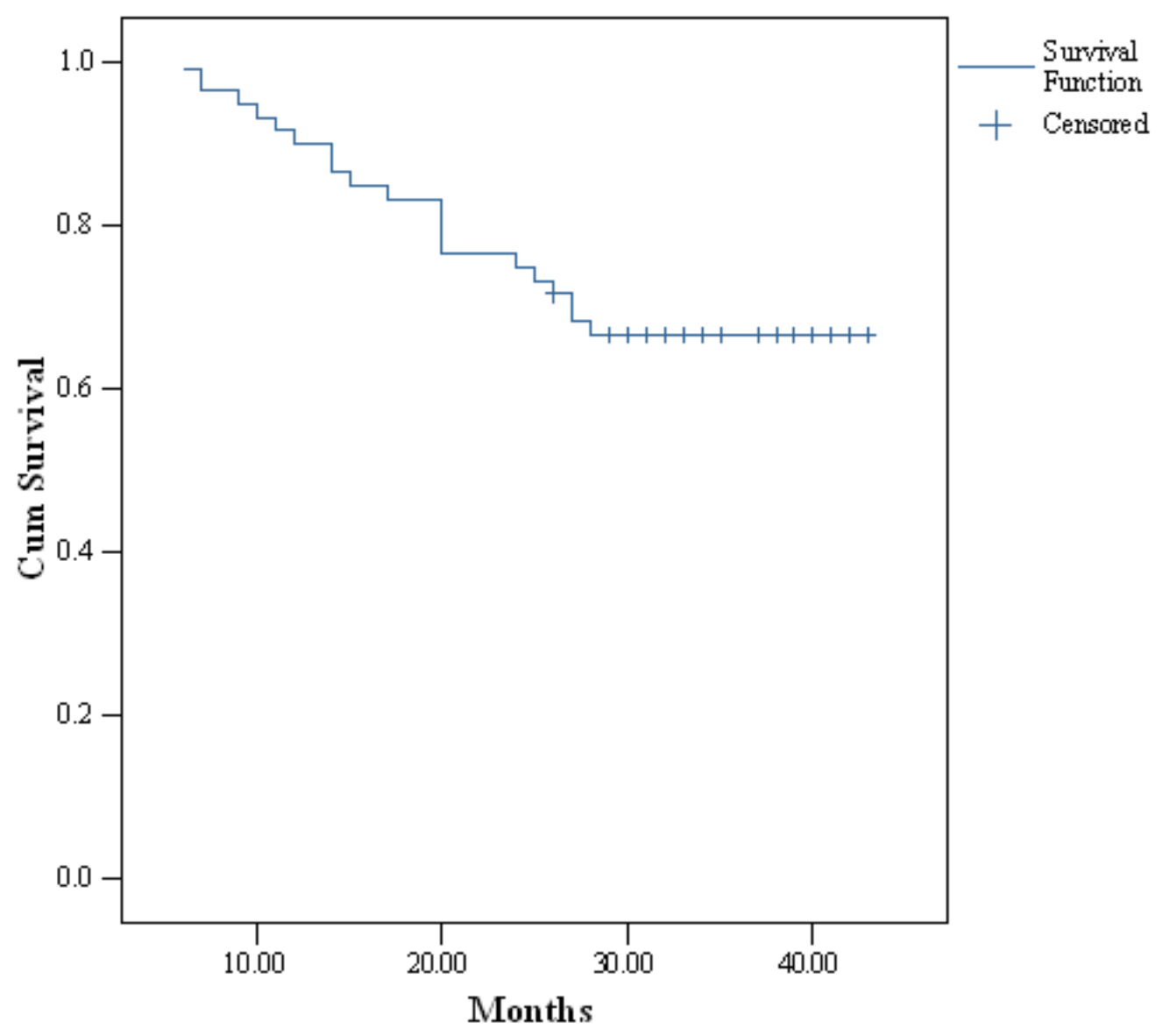

Figure 1 - Overall survival of hormone resistant prostate cancer patients treated with low dose metronomic oral cyclophosphamide. 